\title{
Promoter methylation of heat shock protein B2 in human esophageal squamous cell carcinoma
}

\author{
XIAOFEI CHANG ${ }^{1}$, KEISHI YAMASHITA ${ }^{2}$, DAVID SIDRANSKY ${ }^{1}$ and MYOUNG SOOK KIM ${ }^{1}$ \\ ${ }^{1}$ Department of Otolaryngology, Head and Neck Cancer Research Division, The Johns Hopkins University, \\ School of Medicine, 1550 Orleans Street, CRB II-5M, Baltimore, MD 21231, USA; \\ ${ }^{2}$ Department of Surgery, Kitasato University Hospital, Sagamihara 228-8555, Kanagawa, Japan
}

Received September 7, 2010; Accepted November 3, 2010

DOI: $10.3892 /$ ijo.2011.918

\begin{abstract}
Hypermethylation of gene promoters and the corresponding loss of gene expression are recognized as a hallmark of human cancer, and DNA methylation has emerged as a promising biomarker for the detection of human esophageal squamous cell carcinoma (ESCC). To identify novel genes methylated in ESCC, we screened 35 candidate genes identified from an oligonucleotide microarray. Among them, the heat shock protein B2 (HSPB2) was methylated in 95.7\% (67/70) of primary ESCCs, whereas no methylation was found in normal esophageal tissues from ESCC patients $(0 \%, 0 / 20)$. RT-PCR analysis revealed that HSPB2 expression was silenced or weakly expressed in most ESCC cell lines, and re-activated by the demethylating agent 5-aza-2'-deoxycytidine. These results indicate that promoter methylation of HSPB2 is one of the causal factors for loss or down-regulation of HSPB2 expression. mRNA expression of HSPB2 in ESCC tissues was significantly down-regulated compared to normal tissues. Our data suggest that promoter methylation of HSPB2 deserves further attention as a novel molecular biomarker in human ESCC.
\end{abstract}

\section{Introduction}

Esophageal cancer is one of the common malignancies with an increasing incidence and a high mortality worldwide $(1,2)$. Most patients with ESCC are diagnosed at an advanced stage, and metastasis to the regional lymph nodes occurs frequently (3). As a consequence, the prognosis of ESCC patients is usually very poor. Finding molecular markers for ESCC early detection is thus one of the most promising research avenues that might improve the survival of patients with ESCC.

Correspondence to: Dr Myoung Sook Kim, Department of Otolaryngology, Head and Neck Cancer Research Division, The Johns Hopkins University, School of Medicine, 1550 Orleans Street, CRB II-5M, Baltimore, MD 21231, USA

E-mail: mkim51@jhmi.edu

Key words: heat shock protein B2, methylation, esophageal squamous cell carcinoma
Heat shock protein B2 (HSPB2) is a member of the small heat shock protein family, expressed in heart and skeletal muscles (4). It is also known as heat shock $27-\mathrm{kDa}$ protein 2 (HSP27), located at chromosome 11q22-q23 which is a frequent target for deletion during the development of many solid tumor types (5). HSPB2 is present in the cytosol as granules in association with the outer membrane components of the mitochondria, and thus plays a protective role for cells from deleterious stresses such as oxidative stress, heat shock, radiation and anti-cancer drugs (4-9). It is co-expressed with B-catenin in the cellular compartment of tumor and cell lines, and also interacts with $\beta$-catenin. The biological activity of HSPB 2 is regulated by phosphorylation of its serines by mitogen-activated protein kinases associated protein kinases (MAPKAPKs) which are activated by phosphorylation of p38 mitogen-activated protein (MAP) kinase $(10,11)$. HSP27 regulates Akt activation (12) and p53 transcriptional activity in doxorubicin-treated fibroblasts and cardiac cells (13). Interestingly, HSP27-overexpression in NIH3T3 cells retards cell migration and invasion, which is correlated with reduced expression of matrix metalloproteinase- 2 and Stat 3 phophorylation (14).

Although it has been reported that small heat shock proteins including HSPB2 participates in regulation of apoptosis and are involved in tumorigenesis $(6,13,15,16)$, the expression of HSPB2 is frequently reduced in patients with ESCC, which is associated significantly with poor prognosis and postoperative patient survival $(17,18)$. Expression of HSPB2 correlates negatively with lymph node metastases (18) and also correlates positively with lymphocyte infiltration (18) whereas inversely with depth of invasion (17). These findings suggest that HSPB2 is a significant prognostic factor for ESCC.

Hypermethylation of gene promoters and the corresponding loss of gene expression are now recognized as a hallmark of human cancer (19). We have intensively investigated methylation of gene promoters in primary tumors and in body fluids for the development of biomarkers in cancer (20-23). Recently, a combination of several methylation markers was reported to be significantly correlated with patient prognosis in esophageal adenocarcinoma (24). We also reported that PGP9.5 and NMDAR2B were independent prognostic markers for ESCC (25-27). In this study, we examined the methylation status of the HSPB2 gene promoter in human ESCC, and 
Table I. PCR primers for bisulfite-sequencing.

Forward

Reverse

\begin{tabular}{llll}
\hline NM_001541 & HSPB2 & & \\
& Region 1 & GAAGAGGAGAAAATGAGGTGTGGAA & AAATAAAAACCCTAAATAAAAACAA \\
Region 2 & AGGGTTTTATTTTAGTTTTTT & AATAATATCTATACCATACCTTCTCC \\
NM_001666 & ARHGAP4 & TTAAAACTCTCACACCTACTC & CCGTTGCGGTTAGTATTTTG \\
NM_153613 & LOC254531 & CTCAACTCCCAAACCCACTC & TTTTGGCGTTTTTGTTTTTT \\
NM_003224 & ARFRP1 & CTCTTCTATCTAACCAAAAC & TTGTTGGGGCGAAAAGGGGG \\
NM_003224 & KCNMA1 & CGAATTACCACCTAATAAA & GGGGGGAGGGGGATGGAGTG \\
NM_000517 & HBA2 & TACAAACTAACTTTCTCCCT & GTTAGGGTTTATGTTTGGGG \\
NM_003004 & SECTM1 & CACGAACTCTCCTAAAAAAATACTCC & AGAGGGAGGGGTTAGGGAGGA \\
NM_007075 & WDRX1 & AAACTTAATCTTAACCCAACCCTCC & GAGTTTAGGTATTGAATGGTATTTA \\
NM_007011 & ABHD2 & TAACCCTCCTCCCCTTTCCC & TCGTTTGTTTATTGGGTAGTTTAGT \\
NM_006159 & NELL2 & CCATCAACTTAACACCTTAAATTTA & GTAAGGAGGAGGGAGGGGT \\
NM_003080 & SMPD2 & CCCACCACCTTCACCTACAC & TAAAGTTGAGGAAAGGTTTAGT \\
NM_007074 & COROA1 & TTCGTACCTACCAAAAAAAAACTAA & GATGTTTTTGTTAAGATAATGAATG \\
NM_006454 & MXD4 & TCTACATCTATCAACATTCT & TTTTAGGTTTTTAAATTGATATATA \\
& DTR & & \\
NM_001945 & (HBEGF) & ATCAATAACCCTACCCACAATCAAA & TCGGGAATAAGGTTTTAGGAGG \\
NM_003380 & VIM & CCATACCCAATCCCAAACCC & AGAGTGGTAGAGGATTGGATTT \\
\hline
\end{tabular}

report for the first time that HSPB2 is frequently methylated in primary ESCC but not in normal esophageal tissues. A specific decrease of HSPB2 transcript in ESCC cell lines and tissue was also observed.

\section{Materials and methods}

Cell lines and tissues. ESCC cell lines were obtained from the Cell Response Center for Biomedical Research Institute, Department of Aging and Cancer, Tohoku University (TE series) and kindly provided by Dr Y. Shimada in the Department of Surgery, Kyoto University (KYSE series). Cells were grown in RPMI-1640 supplemented with $10 \%$ fetal bovine serum. Twenty pairs of ESCC and normal esophagus tissues (patient no. 1-20) were obtained from the Gastroenterology Division, Department of Medicine, University of Maryland. Fifty cases of primary ESCC with clinical features, and cDNAs prepared from six ESCC and one normal esophageal tissue were obtained from patients who underwent surgery at the Medical Institute of Bioregulation Hospital, Kyushu University and the Saitama Cancer Center $(26,27)$. Five normal esophageal epithelial tissues (NN) were obtained from patients without cancer at the Department of Pathology, The Johns Hopkins University.

Bisulfite-sequencing. Bisulfite-modified genomic DNA was amplified by PCR as described (26). Primers were designed in the promoter proximal to transcription start site (TSS), and primer sequences are shown in Table I.

The criteria to determine methylation in cell lines and tissues. Bisulfite-sequencing was based on nucleotide sequences in electropherograms, and the criteria to determine methylation positivity was reported previously (28). When methylated CpG was found in $>30 \%$ of total CpGs in an amplified PCR product, it was considered as methylation-positive.

5-aza-dC and TSA treatment and RT-PCR. Treatment with 5-aza-2'-deocycytidine (5-aza-dC) and/or trichostatin A (TSA), and RNA extraction and cDNA synthesis were performed as described (26). HSPB2 primers were 5'-ATGTCGGGCCG CTCAGTGCC-3' (forward) and 5'-GTCACCTCGTCTGGG GTAAA-3' (reverse). GAPDH primers were 5'-CAACTAC ATGGTTTACATGTTC-3' (forward) and 5'-GCCAGTGGA CTCCACGAC-3' (reverse). PCR products were gel-extracted and sequenced to verify true expression of the gene.

Quantitative RT-PCR. cDNAs from an ESCC patient and from a patient without cancer (NN) were purchased from BioChain Institute, Inc. (Hayward, CA). A pair of normal (PN) and tumor cDNA (PT) was obtained from patients who underwent surgery at the Medical Institute of Bioregulation Hospital, Kyushu University. Real-time RT-PCR was performed as described previously (28). 


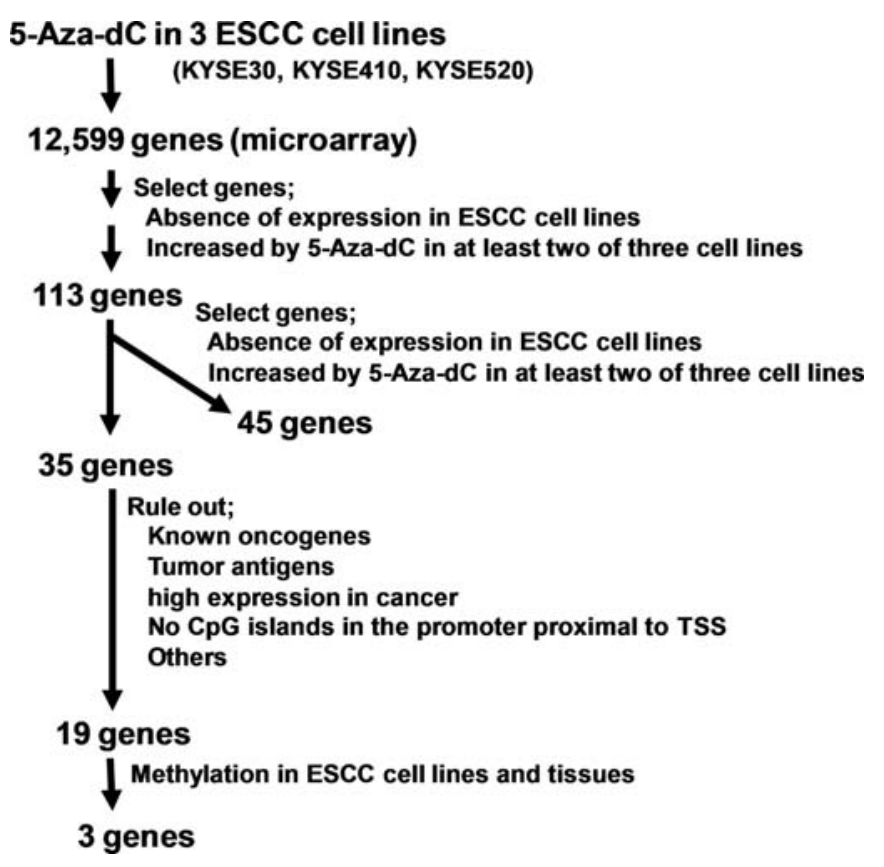

Figure 1. Flowchart for selection of candidate genes in ESCC by pharmacologic unmasking and microarray analysis. We identified candidatemethylated genes which were epigenetically silenced in ESCC cell lines using pharmacologic reversal of methylation followed by microarray analysis.

\section{Results}

After ruling out presumed background genes we prioritized candidate genes according to the microarray data described previously (26). We randomly selected 35 genes and further diminished the number of candidate genes to 19 genes by excluding known oncogenes, tumor antigens, genes with high expression in tumor, genes with no $\mathrm{CpG}$ islands near the transcription start site (TSS), and genes whose methylation status was already known (Fig. 1). We examined promoter methylation status of these 19 genes by bisulfite-sequencing in three ESCC cell lines (KYSE30, KYSE410, KYSE520) and four tumor tissue samples (PT). To rule out genes methylated in normal tissues, we also examined these genes in 4 normal adjacent esophageal mucosa (PN) samples collected from ESCC patients. As a result, we found that heat shock protein B2 (HSPB2), Rho GTPase activating protein 4 (ARHGAP4), $\alpha 2$ hemoglobin (HBA2), a hypothetical protein (LOC254531), potassium large conductance calciumactivated channel (KCNMA1) and ADP-ribosylation factor related protein 1 (ARFRP1) were methylated in ESCC cell lines and tissues, but not in 4 normal esophageal tissues (Table II). Epsin (HPN) and type 1-troponin I (TNNI1) were found to be methylated in normal esophageal mucosa and thus were excluded. The other eleven genes were not methylated in either ESCC cell lines or primary tissues.

Table II. Methylation profiles of genes in ESCC cel lines and tissues.

\begin{tabular}{|c|c|c|c|c|c|c|c|c|c|c|c|}
\hline & \multicolumn{3}{|c|}{ Cell lines } & \multicolumn{4}{|c|}{ PT } & \multicolumn{4}{|c|}{ PN } \\
\hline & KYSE30 & KYSE410 & KYSE520 & $\mathrm{T} 5$ & T6 & $\mathrm{T} 7$ & $\mathrm{~T} 8$ & $\mathrm{~N} 2$ & N4 & N5 & N6 \\
\hline HSPB2 & M & M & M & M & $\mathrm{M}$ & $\mathrm{M}$ & M & $\mathrm{U}$ & $\mathrm{U}$ & $\mathrm{U}$ & $\mathrm{U}$ \\
\hline LOC 254531 & & & M & M & $\mathrm{M}$ & $\mathrm{M}$ & $\mathrm{M}$ & $\mathrm{U}$ & $\mathrm{U}$ & $\mathrm{U}$ & $\mathrm{U}$ \\
\hline ARFRP1 & M & M & & M & $\mathrm{M}$ & $\mathrm{M}$ & M & & & $\mathrm{U}$ & $\mathrm{U}$ \\
\hline KCNMA1 & & & M & $\mathrm{M}$ & M & $\mathrm{M}$ & M & & $\mathrm{U}$ & & $\mathrm{U}$ \\
\hline ARHGAP4 & M & M & M & M & $\mathrm{M}$ & $\mathrm{M}$ & M & $\mathrm{U}$ & $\mathrm{U}$ & $\mathrm{U}$ & $\mathrm{U}$ \\
\hline HBA2 & M & $\mathrm{M}$ & $\mathrm{M}$ & $\mathrm{M}$ & $\mathrm{U}$ & $\mathrm{U}$ & M & $\mathrm{U}$ & $\mathrm{U}$ & $\mathrm{U}$ & $\mathrm{U}$ \\
\hline HPN & M & $\mathrm{M}$ & $\mathrm{M}$ & & & & & $\mathrm{M}$ & $\mathrm{M}$ & $\mathrm{M}$ & $\mathrm{M}$ \\
\hline TNNI1 & M & M & M & & & & & M & $\mathrm{M}$ & M & M \\
\hline DFKZP564008232 & $\mathrm{U}$ & $\mathrm{U}$ & & $\mathrm{U}$ & $\mathrm{U}$ & $\mathrm{U}$ & $\mathrm{U}$ & $\mathrm{U}$ & $\mathrm{U}$ & $\mathrm{U}$ & $\mathrm{U}$ \\
\hline SECTM1 & $\mathrm{U}$ & $\mathrm{U}$ & $\mathrm{U}$ & $\mathrm{U}$ & & $\mathrm{U}$ & $\mathrm{U}$ & $\mathrm{U}$ & $\mathrm{U}$ & $\mathrm{U}$ & $\mathrm{U}$ \\
\hline WDRX1 & $\mathrm{U}$ & $\mathrm{U}$ & $\mathrm{U}$ & $\mathrm{U}$ & $\mathrm{U}$ & $\mathrm{U}$ & & $\mathrm{U}$ & $\mathrm{U}$ & $\mathrm{U}$ & $\mathrm{U}$ \\
\hline ABHD2 & $\mathrm{U}$ & $\mathrm{U}$ & $\mathrm{U}$ & $\mathrm{U}$ & & $\mathrm{U}$ & $\mathrm{U}$ & $\mathrm{U}$ & $\mathrm{U}$ & $\mathrm{U}$ & $\mathrm{U}$ \\
\hline NELL2 & $\mathrm{U}$ & $\mathrm{U}$ & $\mathrm{U}$ & $\mathrm{U}$ & $\mathrm{U}$ & $\mathrm{U}$ & $\mathrm{U}$ & $\mathrm{U}$ & $\mathrm{U}$ & $\mathrm{U}$ & $\mathrm{U}$ \\
\hline SMPD2 & $\mathrm{U}$ & $\mathrm{U}$ & $\mathrm{U}$ & $\mathrm{U}$ & $\mathrm{U}$ & $\mathrm{U}$ & $\mathrm{U}$ & $\mathrm{U}$ & $\mathrm{U}$ & $\mathrm{U}$ & $\mathrm{U}$ \\
\hline COROA1 & $\mathrm{U}$ & $\mathrm{U}$ & $\mathrm{U}$ & $\mathrm{U}$ & & & & $\mathrm{U}$ & $\mathrm{U}$ & $\mathrm{U}$ & $\mathrm{U}$ \\
\hline MXD4 & $\mathrm{U}$ & $\mathrm{U}$ & $\mathrm{U}$ & $\mathrm{U}$ & $\mathrm{U}$ & $\mathrm{U}$ & $\mathrm{U}$ & $\mathrm{U}$ & $\mathrm{U}$ & $\mathrm{U}$ & $\mathrm{U}$ \\
\hline DTR & $\mathrm{U}$ & $\mathrm{U}$ & $\mathrm{U}$ & $\mathrm{U}$ & & $\mathrm{U}$ & & $\mathrm{U}$ & $\mathrm{U}$ & $\mathrm{U}$ & $\mathrm{U}$ \\
\hline VIM & $\mathrm{U}$ & $\mathrm{U}$ & $\mathrm{U}$ & $\mathrm{U}$ & $\mathrm{U}$ & $\mathrm{U}$ & $\mathrm{U}$ & $\mathrm{U}$ & $\mathrm{U}$ & $\mathrm{U}$ & $\mathrm{U}$ \\
\hline IGFBP7 & $\mathrm{U}$ & $\mathrm{U}$ & $\mathrm{U}$ & & & & & $\mathrm{U}$ & $\mathrm{U}$ & $\mathrm{U}$ & $\mathrm{U}$ \\
\hline
\end{tabular}

M, methylated; U, unmethylated. PN, normal esophageal mucosa collected from ESCC patients; PT, ESCC tissues. Note, HSPB2 methylaton was examined in the region 1 of the promoter. 


\begin{tabular}{|c|c|c|c|c|c|c|c|c|c|c|c|c|c|c|c|c|c|c|c|c|}
\hline \multirow[t]{2}{*}{$\mathbf{A}$} & \multicolumn{20}{|c|}{ PT } \\
\hline & T1 & $\mathrm{T} 2$ & $T 3$ & T4 & T5 & T6 & $\mathrm{T7}$ & T8 & T9 & T10 & T11 & $\mathrm{T} 12$ & T13 & T14 & T15 & T16 & T17 & T18 & T19 & $\mathrm{T} 20$ \\
\hline HSPB2 & $M$ & M & $\mathbf{M}$ & M & M & $M$ & M & $M$ & M & $U$ & $M$ & M & M & M & M & M & M & M & $U$ & M \\
\hline LOC254531 & $M$ & M & $\mathbf{M}$ & M & $\mathbf{M}$ & $M$ & M & $\mathbf{M}$ & M & $M$ & M & M & $M$ & $M$ & $M$ & M & M & $M$ & $u$ & M \\
\hline ARHGAP4 & $M$ & M & $\mathbf{M}$ & M & $\mathbf{M}$ & $M$ & M & $U$ & M & $M$ & $M$ & $M$ & $M$ & $M$ & $M$ & M & $U$ & $M$ & $U$ & M \\
\hline ARFRP1 & $\mathrm{u}$ & $\mathrm{U}$ & $U$ & $U$ & $M$ & $M$ & $M$ & $M$ & $\mathrm{U}$ & $\mathrm{u}$ & M & u & $u$ & $u$ & u & $u$ & M & $u$ & M & $u$ \\
\hline KCNMA1 & $u$ & M & $U$ & $u$ & $M$ & $M$ & M & $M$ & $U$ & $u$ & $M$ & $U$ & $M$ & $u$ & $M$ & $M$ & $M$ & $u$ & $u$ & $u$ \\
\hline \multirow[t]{3}{*}{ HBA2 } & u & $U$ & $M$ & $U$ & $M$ & $u$ & $U$ & $M$ & $U$ & $u$ & $u$ & $M$ & $M$ & $u$ & $M$ & $M$ & $M$ & $u$ & $u$ & $u$ \\
\hline & \multicolumn{20}{|c|}{ PN } \\
\hline & N1 & N2 & N3 & N4 & N5 & N6 & N7 & N8 & N9 & N10 & N11 & N12 & N13 & N14 & N15 & N16 & N17 & N18 & N19 & $\mathrm{N} 20$ \\
\hline HSPB2 & $u$ & U & $U$ & U & U & $u$ & U & $U$ & U & $u$ & u & U & $u$ & $u$ & $u$ & $u$ & u & $u$ & $u$ & $u$ \\
\hline LOC254531 & U & $u$ & $\mathbf{M}$ & $u$ & $U$ & $u$ & $U$ & $U$ & U & $u$ & $u$ & $u$ & $u$ & M & $u$ & $u$ & $u$ & $u$ & M & $u$ \\
\hline ARHGAP4 & $\mathrm{U}$ & $U$ & $M$ & $U$ & $\mathrm{U}$ & $u$ & $U$ & $\mathrm{U}$ & $\mathrm{U}$ & $U$ & $M$ & $U$ & $\mathrm{U}$ & $\mathrm{U}$ & $\mathrm{U}$ & $U$ & $U$ & $M$ & $\mathrm{U}$ & $M$ \\
\hline
\end{tabular}

B
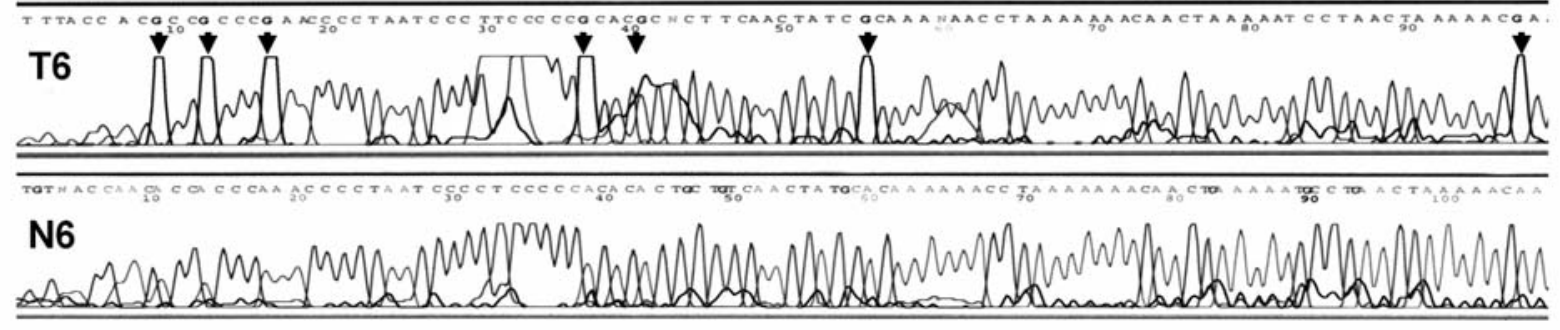

C

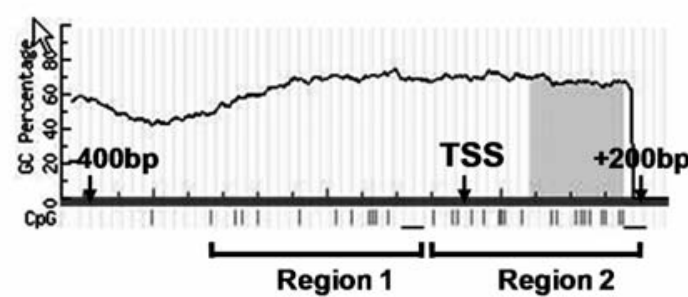

CTGCCACCGAAGAGGAGAAAATGAGGTGTGGAAACGTGCAAAC $\mathbf{2}$ ' TGTGAGGGTCTCAGCGAGGCCTCTTCATGCCCCAGGCACCACCAGCT CCCCCTCCCCAGCTCG̈TTCCCAGCCAGGACCCTCAGCTGTCCTCTCA GgCCCCCTGCGACAGCTGAaGAGTGTGCGGGGGAGgGGACTAGGG $\frac{\mathbf{7}}{\text { GTCCGG G }^{\mathbf{8}} \mathbf{9}} \mathbf{9}$ CGCTGGTCACACCCTCGTT $\frac{10}{\text { GCTCTCACTCAGGGCCC }}$ CCACTCCCAGCCCCTCCCCCCCCAAGAGGCTCGGCACTATTTTGGGT $]$

\begin{tabular}{ll|l|l|l|l|l|l|l|l|l|l|l|l|l|l|l|l|l|c|c|}
$\mathbf{D}$ & $\mathbf{1}$ \\
$\mathbf{D}$ & $\mathrm{PT}$ \\
\hline & $\mathrm{MN}$ & $\mathrm{M}$ & $\mathrm{M}$ & $\mathrm{M}$ & $\mathrm{M}$ & $\mathrm{M}$ & $\mathrm{M}$ & $\mathrm{M}$ & $\mathrm{M}$ & $\mathrm{M}$ & $\mathrm{M}$ & $\mathrm{M}$ & $\mathrm{M}$ & $\mathrm{M}$ & $\mathrm{M}$ & $\mathrm{M}$ & $\mathrm{M}$ & $\mathrm{M}$ & $\mathrm{M}$ \\
\hline & $\mathrm{M}$ & $\mathrm{M}$ & $\mathrm{U}$ & $\mathrm{M}$ & $\mathrm{U}$ & $\mathrm{M}$ & $\mathrm{M}$ & $\mathrm{M}$ & $\mathrm{U}$ & $\mathrm{U}$ & $\mathrm{U}$ & $\mathrm{M}$ & $\mathrm{M}$ & $\mathrm{M}$ & $\mathrm{M}$ & $\mathrm{M}$ & $\mathrm{U}$ & $\mathrm{M}$ & $\mathrm{M}$ \\
\hline
\end{tabular}

Figure 2. Methylation status of HSPB2 in ESCC and matched normal esophageal tissues. (A) Methylation status of six candidate genes selected in the pilot study were examined in 20 pairs of ESCC (PT) and matched normal esophageal tissues (PN) by bisulfite-sequencing (upper). Bisulfite-sequencing results in region 1 of the HSPB2 promoter. Grey square with M, methylation; white square with U, absence of methylation. (B) Representative sequencing results of HSPB2. Arrowhead, all guanines present after sequencing that are complementary to methyl cytosines on the opposite DNA strand. (C) CpG methylation of the HSPB2 promoter was examined in two regions (1 and 2). The CG sequences of region 1 are underlined and numbered, and the PCR-amplified region is indicated as F1 (forward) and R1 (reverse) primers. (D) Bisulfite-sequencing results in region 2. Grey square with M, methylation; white square with U, absence of methylation.

Next, we examined the methylation status of the above 6 candidate genes in 20 ESCC tumor tissue samples (PT). ARFRP1 (35\%, 7/20), KCNMA1 $(45 \%, 9 / 20)$ and HBA2 $(40 \%, 8 / 20)$ were methylated with relatively low frequency, whereas HSPB2 $(90 \%, 18 / 20)$, LOC254531 (95\%, 19/20), and ARHGAP4 $(85 \%, 17 / 20)$ were methylated in ESCC with high frequency (Fig. 2A, upper). In addition, HSPB2, LOC254531 and ARHGAP4 were methylated in $<20 \%$ of the 20 normal matched esophageal tissues, indicating that the methylation was cancer-specific (Fig. 2A, lower). Among the three genes, HSPB2 was selected for further analysis due to the absence of methylation in all 20 matched normal appearing esophageal tissues (PN) (0\%). Representative sequencing results of HSPB2 are shown in Fig. 2B. It is noteworthy that the HSPB2 promoter region we examined (region 1) contained no $\mathrm{CpG}$ island. We have investigated the region that contained one $\mathrm{CpG}$ island (region 2) and found a higher methylation frequency in the normal appearing esophageal tissues (Fig. 2C and D). These data suggested normal and malignant esophageal tissues were distinguished more significantly by the methylation of HSPB2 in region 1 than in region 2.

We numbered the ten CpGs in region 1 of the HSPB2 promoter, and the methylation status of each individual $\mathrm{CpG}$ was mapped. Five normal esophageal tissues from patients without cancer (Eso NN) were also included for comparison. Our data showed that none of the CpGs were methylated in Eso NN (Fig. 3A). CpGs in positions 1-7 were found to be commonly methylated in 12 ESCC cell lines and 18 ESCC tissues (PT), (Fig. 3B and C). In matched normal esophageal tissues (PN), only one methylated $\mathrm{CpG}$ was found (position 5 or 6) in 7 out of 20 cases. We further examined HSPB2 promoter methylation in $50 \mathrm{ESCC}$, and found a methylation frequency of 98\% (49/50). Taken together, the overall HSPB2 methylation was observed in a total of 67 out of 70 ESCC cases $(95.7 \%, \mathrm{P}=0.02)$.

To examine the transcriptional level of HSPB2, RT-PCR was performed using primers specific for HSPB2. Weak expression of HSPB2 was observed in the TE series, and no 
A

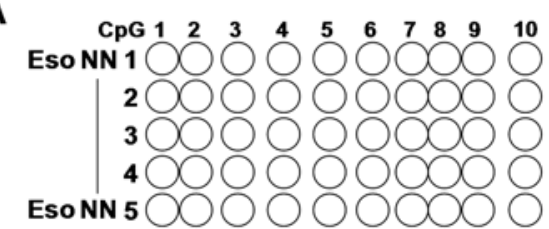

B

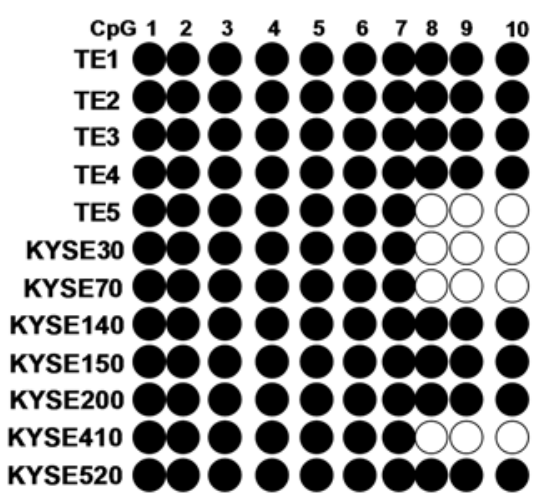

C

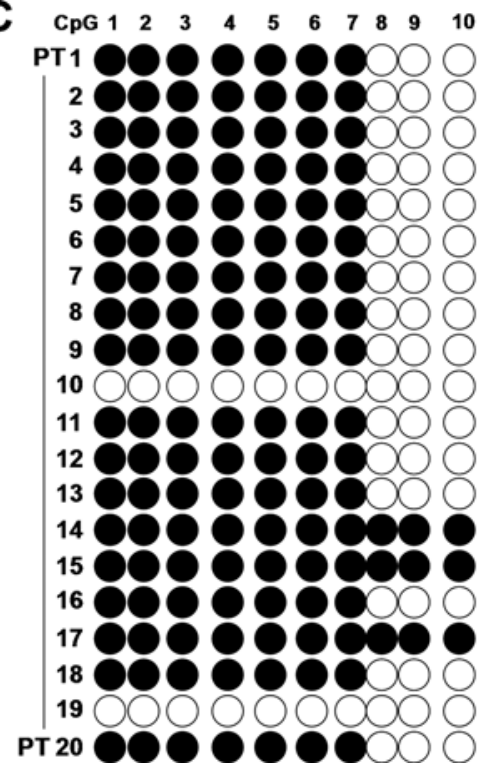

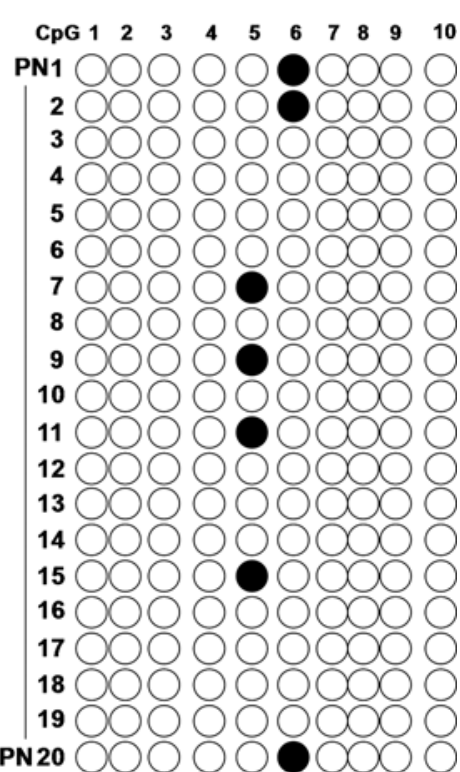

Figure 3. Individual CpG methylation map of the HSPB2 promoter. Individual CpG site methylation was mapped in five normal esophageal tissues from patients without cancer (Eso NN) (A), 12 ESCC cell lines (B), and 20 pairs of ESCC (PT) and matched normal esophageal tissues (PN) (C). Black circle, methylayed $\mathrm{CpG}$; white circle, unmethylated $\mathrm{CpG}$.

A

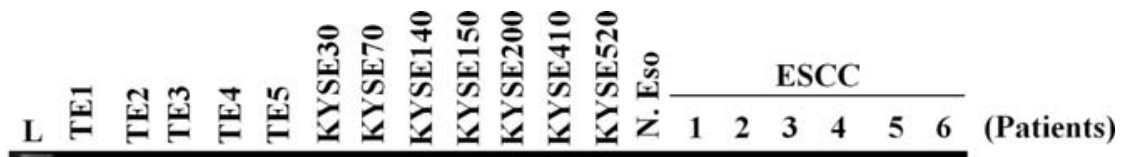

\section{HSPB2}

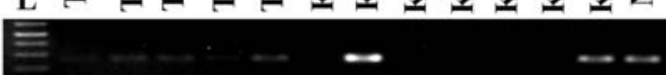

GAPDH

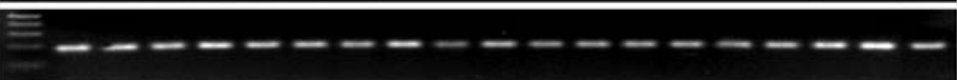

B

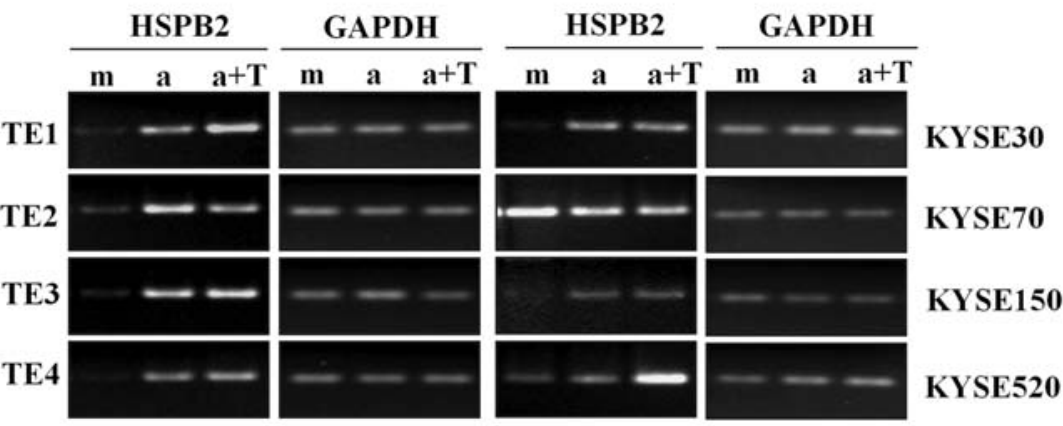

C

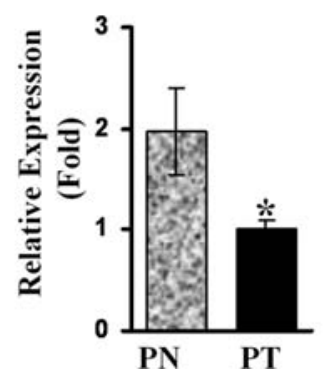

D

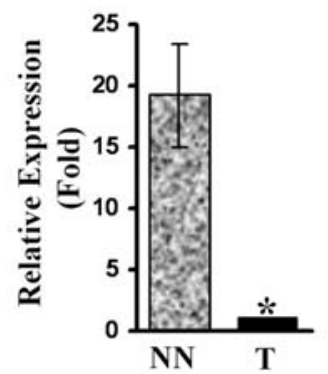

Figure 4. Promoter hypermethylation downregulates expression of HSPB2 in ESCC cell lines. (A) RT-PCR analysis for HSPB2 expression in cell lines and tissues. GAPDH was used as a loading control. L, $1 \mathrm{~kb}$ Plus DNA ladder. (B) Reactivation of HSPB2 by $5 \mu \mathrm{M} 5$-Aza-dC and/or TSA treatment. m, mock treatment; a, 5-Aza-dC treatment; a+T, 5-Aza-dC + TSA treatment. (C) HSPB2 expression in a pair of PN and PT cDNA prepared from an ESCC patient by real-time PCR. (D) HSPB2 expression in patients with ESCC (T) or without cancer (NN) by real-time PCR. Relative expression (fold) was calculated by comparing the ratios of mRNA expression of HSPB2 to an internal control gene, GAPDH. Experiments were done in duplicate, and values indicate the means \pm SD. ${ }^{*} \mathrm{P}<0.05$ (t-test). 
detectable level of HSPB2 was observed in most of the KYSE series (Fig. 4A). Unexpectedly, HSPB2 was expressed in KYSE70 and KYSE520, two cell lines which harbored HSPB2 promoter methylation. However, the mRNA level of HSPB2 was reactivated by treatment with 5 -aza-dC, and/or trichostatin A (TSA) in all ESCC cell lines (Fig. 4B, and data not shown), except for the KYSE70 cells where reactivation was minimal. These results indicate that the transcriptional level of HSPB2 is regulated, although not in all cases, by promoter methylation. HSPB2 expression was also examined in cDNA prepared from one normal esophagus (N. Eso) and six ESCC tissues (patients 1-6) by RT-PCR (Fig. 4A, right lanes). HSPB2 expression was observed only in normal esophageal cDNA. In addition, real-time RT-PCR analysis performed in a pair of normal and tumor cDNA prepared from an ESCC patient showed that HSPB2 expression in PN was twice higher than in PT (Fig. 4C, P<0.05). Moreover, the level of HSPB2 in normal cDNA prepared from a patient without cancer was twenty times higher than in tumor cDNA from an ESCC patient (Fig. 4D, $\mathrm{P}<0.05$ ). These results suggest that HSPB2 expression is markedly down-regulated in ESCC.

\section{Discussion}

Promoter methylation is a promising type of molecular marker in human cancer (29). In previous studies, we have established a set of strategies to screen new potential methylation markers in different types of tumors $(26,28,30)$. We undertook pharmacological unmasking of ESCC cell lines with 5-aza-dC and TSA followed by microarray analysis to comprehensively identify epigenetically inactivated genes in ESCC (26). Among the large number of genes identified by the microarray analysis, we selected in this study candidate genes of which expression was initially absent and then reactivated by treatment with 5-aza-dC. We evaluated the methylation status of the promoter regions in 19 genes by bisulfite-sequencing, and discovered that three genes (HSPB2, LOC254531 and ARHGAP4) harbored methylation in $>80 \%$ of ESCC tissues.

Based on the differential methylation status of these three genes in human ESCC and normal tissue, we selected HSPB2 to further evaluate its potential as a biomarker in ESCC. The high methylation frequency in ESCC $(95.7 \%, 67 / 70)$ strongly supports that HSPB2 is a cancer-specific methylation marker in human ESCC. To the best of our knowledge, this is the first report on HSPB2 promoter methylation in human cancers. Although the region we examined contains no $\mathrm{CpG}$ island, discrimination of ESCC from matched normal esophageal tissues was significant for methylation in this region.

HSPB2 belongs to a sub-family of small heat shock proteins that display an enhanced expression in response to various stresses (e.g., heat shock) (4). HSPB2 with other members of HSP family (e.g., Hsp90, Hsp70) has been implicated in tumorigenesis, which may be related to their ability to protect cells against stress-activated programmed cell death (31). The enhanced expression of HSPB2 has been associated with poor prognosis in several tumor types (32-39), however, it also showed a positive correlation with favorable prognosis in many types of cancers $(17,18,40-45)$. These differences seen in the value of HSPB2 as a prognostic factor are thought to be at least partly due to the inherent differences in other biological and molecular features of each cancer type.

Silencing of small heat shock proteins (HSP) has been reported previously; expression of $\alpha B$-crystallin (CRYAB), a low-molecular-weight HSP, was down-regulated in highly malignant anaplastic thyroid carcinomas (ATC) (46) and ESCC. In addition, $\alpha \mathrm{B}$-crystallin and Hsp25, the mouse homolog of human HSP27, were not expressed in the P388 murine leukemic cell line (47), indicating a defect or a block in expression of these genes. Interestingly, promoter methylation of the $\alpha \mathrm{B}$-crystallin gene occurred in ATC (46) and ESCC cell lines (48), but it was not clear whether DNAmethylation silenced the expression of Hsp25 and $\alpha \mathrm{B}$ crystallin genes in $\mathrm{P} 388$ cells since no reactivation was observed in mRNA levels after 5-azacytidine treatment (47).

In our results, expression of the HSPB2 gene was clearly repressed at the mRNA level in most ESCC cell lines tested, but reactivated after 5-aza-dC and/or TSA treatment, indicating regulation of gene expression by promoter methylation of HSPB2 in ESCC cell lines. Real-time RT-PCR analysis revealed that the mRNA level of HSPB2 was $>20$ times higher in normal esophageal tissue than in ESCC. In addition, previous studies reported that protein expression of HSPB2 was frequently reduced in patients with $\operatorname{ESCC}(17,18)$. Taken together, these results suggest that promoter methylation may play an important role in down-regulation of HSPB2 expression in ESCC.

In conclusion, we have found that HSPB2 was methylated in human ESCC in a cancer-specific manner. HSPB2 promoter methylation thus deserves further attention as a potential diagnostic DNA marker in human ESCC. In addition, the biological and molecular consequences of HSPB2 downregulation in ESCC carcinogenesis remain to be elucidated.

\section{Acknowledgements}

This study was supported by National Cancer Institute (U01CA84986), Oncomethylome Sciences, and the Flight Attendant Medical Research Institute (Myoung S. Kim). Conflict of interest: Under a licensing agreement between Oncomethylome Sciences and the Johns Hopkins University, D. Sidransky is entitled to a share of royalties received by the University upon sales of any products described in this article. D. Sidransky owns Oncomethylome Sciences SA stock, which is subject to certain restrictions under University policy. D. Sidransky is a paid consultant to Oncomethylome Sciences SA, and is a paid member of the company's Scientific Advisory Board. The Johns Hopkins University in accordance with its conflict of interest policies is managing the terms of this agreement.

\section{References}

1. WHO: The World Health Report, 1997

2. Pisani P, Parkin DM, Bray F and Ferlay J: Estimates of the worldwide mortality from 25 cancers in 1990. Int J Cancer 83: 18-29, 1999.

3. Goseki N, Koike M and Yoshida M: Histopathologic characteristics of early stage esophageal carcinoma. A comparative study with gastric carcinoma. Cancer 69: 1088-1093, 1992.

4. Nakagawa M, Tsujimoto N, Nakagawa H, Iwaki T, Fukumaki Y and Iwaki A: Association of HSPB2, a member of the small heat shock protein family, with mitochondria. Exp Cell Res 271: $161-168,2001$ 
5. Baysal BE, Willett-Brozick JE, Taschner PE, Dauwerse JG, Devilee P and Devlin B: A high-resolution integrated map spanning the SDHD gene at 11q23: a 1.1-Mb BAC contig, a partial transcript map and 15 new repeat polymorphisms in a tumour-suppressor region. Eur J Hum Genet 9: 121-129, 2001.

6. Aloy MT, Hadchity E, Bionda C, et al: Protective role of Hsp27 protein against gamma radiation-induced apoptosis and radiosensitization effects of Hsp27 gene silencing in different human tumor cells. Int J Radiat Oncol Biol Phys 70: 543-553, 2008.

7. Fortin A, Raybaud-Diogene H, Tetu B, Huot J, Blondeau L and Landry J: Markers of neck failure in oral cavity and oropharyngeal carcinomas treated with radiotherapy. Head Neck 23: $87-93,2001$

8. Oesterreich S, Weng CN, Qiu M, Hilsenbeck SG, Osborne CK and Fuqua SA: The small heat shock protein hsp27 is correlated with growth and drug resistance in human breast cancer cell lines. Cancer Res 53: 4443-4448, 1993.

9. Yoshida M, Niwa M, Ishisaki A, et al: Methotrexate enhances prostaglandin D2-stimulated heat shock protein 27 induction in osteoblasts. Prostaglandins Leukot Essent Fatty Acids 71: 351-362, 2004

10. Rouse J, Cohen P, Trigon S, et al: A novel kinase cascade triggered by stress and heat shock that stimulates MAPKAP kinase-2 and phosphorylation of the small heat shock proteins. Cell 78: 1027-1037, 1994

11. Stokoe D, Engel K, Campbell DG, Cohen P and Gaestel M: Identification of MAPKAP kinase 2 as a major enzyme responsible for the phosphorylation of the small mammalian heat shock proteins. FEBS Lett 313: 307-313, 1992

12. Wu R, Kausar H, Johnson P, Montoya-Durango DE, Merchant M and Rane MJ: Hsp27 regulates Akt activation and polymorphonuclear leukocyte apoptosis by scaffolding MK2 to Akt signal complex. J Biol Chem 282: 21598-21608, 2007

13. Venkatakrishnan CD, Dunsmore K, Wong H, et al: HSP27 regulates p53 transcriptional activity in doxorubicin-treated fibroblasts and cardiac H9c2 cells: p21 upregulation and G2/M phase cell cycle arrest. Am J Physiol Heart Circ Physiol 294: H1736-H1744, 2008.

14. Lee JW, Kwak HJ, Lee JJ, et al: HSP27 regulates cell adhesion and invasion via modulation of focal adhesion kinase and MMP-2 expression. Eur J Cell Biol 87: 377-387, 2008.

15. Laskowska E: [Small heat shock proteins-role in apoptosis, cancerogenesis and diseases associated with protein aggregation]. Postepy Biochem 53: 19-26, 2007.

16. Hensbergen P, Alewijnse A, Kempenaar J, et al: Proteomic profiling identifies an UV-induced activation of cofilin-1 and destrin in human epidermis. J Invest Dermatol 124: 818-824, 2005.

17. Nakajima M, Kuwano H, Miyazaki T, Masuda N and Kato $\mathrm{H}$ : Significant correlation between expression of heat shock proteins 27,70 and lymphocyte infiltration in esophageal squamous cell carcinoma. Cancer Lett 178: 99-106, 2002.

18. Kawanishi K, Shiozaki H, Doki Y, et al: Prognostic significance of heat shock proteins 27 and 70 in patients with squamous cell carcinoma of the esophagus. Cancer 85: 1649-1657, 1999.

19. Baylin SB, Herman JG, Graff JR, Vertino PM and Issa JP: Alterations in DNA methylation: a fundamental aspect of neoplasia. Adv Cancer Res 72: 141-196, 1998.

20. Ishiuchi S, Tsuzuki K, Yoshida Y, et al: Blockage of $\mathrm{Ca}(2+)$ permeable AMPA receptors suppresses migration and induces apoptosis in human glioblastoma cells. Nat Med 8: 971-978, 2002.

21. Rush LJ, Raval A, Funchain P, et al: Epigenetic profiling in chronic lymphocytic leukemia reveals novel methylation targets. Cancer Res 64: 2424-2433, 2004.

22. Sinclair PB, Sorour A, Martineau M, et al: A fluorescence in situ hybridization map of $6 \mathrm{q}$ deletions in acute lymphocytic leukemia: identification and analysis of a candidate tumor suppressor gene. Cancer Res 64: 4089-4098, 2004.

23. Mandard AM, Hainaut P and Hollstein M: Genetic steps in the development of squamous cell carcinoma of the esophagus. Mutat Res 462: 335-342, 2000.

24. Sakimura K, Kutsuwada T, Ito I, et al: Reduced hippocampal LTP and spatial learning in mice lacking NMDA receptor epsilon 1 subunit. Nature 373: 151-155, 1995.

25. Mandelker DL, Yamashita K, Tokumaru Y, et al: PGP9.5 promoter methylation is an independent prognostic factor for esophageal squamous cell carcinoma. Cancer Res 65: 4963-4968, 2005.
26. Kim MS, Yamashita K, Baek JH, et al: N-methyl-D-aspartate receptor type $2 \mathrm{~B}$ is epigenetically inactivated and exhibits tumor-suppressive activity in human esophageal cancer. Cancer Res 66: 3409-3418, 2006

27. Kim MS, Yamashita K, Chae YK, et al: A promoter methylation pattern in the N-methyl-D-aspartate receptor $2 \mathrm{~B}$ gene predicts poor prognosis in esophageal squamous cell carcinoma. Clin Cancer Res 13: 6658-6665, 2007.

28. Kim MS, Louwagie J, Carvalho B, et al: Promoter DNA methylation of oncostatin $m$ receptor-beta as a novel diagnostic and therapeutic marker in colon cancer. PLoS One 4: e6555, 2009.

29. Sidransky D: Emerging molecular markers of cancer. Nat Rev Cancer 2: 210-219, 2002.

30. Yamashita K, Upadhyay S, Osada M, et al: Pharmacologic unmasking of epigenetically silenced tumor suppressor genes in esophageal squamous cell carcinoma. Cancer Cell 2: 485-495, 2002.

31. Calderwood SK, Khaleque MA, Sawyer DB and Ciocca DR: Heat shock proteins in cancer: chaperones of tumorigenesis Trends Biochem Sci 31: 164-172, 2006.

32. Cornford PA, Dodson AR, Parsons KF, et al: Heat shock protein expression independently predicts clinical outcome in prostate cancer. Cancer Res 60: 7099-7105, 2000

33. El-Meghawry El-Kenawy A, El-Kott AF and Hasan MS: Heat shock protein expression independently predicts survival outcome in schistosomiasis-associated urinary bladder cancer. Int J Biol Markers 23: 214-218, 2008.

34. Glaessgen A, Jonmarker S, Lindberg A, et al: Heat shock proteins 27,60 and 70 as prognostic markers of prostate cancer. APMIS 116: 888-895, 2008

35. Kapranos N, Kominea A, Konstantinopoulos PA, et al: Expression of the $27-\mathrm{kDa}$ heat shock protein (HSP27) in gastric carcinomas and adjacent normal, metaplastic, and dysplastic gastric mucosa, and its prognostic significance. J Cancer Res Clin Oncol 128: 426-432, 2002.

36. King KL, Li AF, Chau GY, et al: Prognostic significance of heat shock protein-27 expression in hepatocellular carcinoma and its relation to histologic grading and survival. Cancer 88: 2464-2470, 2000

37. Romanucci M, Marinelli A, Sarli G and Della Salda L: Heat shock protein expression in canine malignant mammary tumours. BMC Cancer 6: 171, 2006.

38. Takeno S, Noguchi T, Kikuchi R, Sato T, Uchida Y and Yokoyama S: Analysis of the survival period in resectable stage IV gastric cancer. Ann Surg Oncol 8: 215-221, 2001.

39. Thanner F, Sutterlin MW, Kapp M, et al: Heat shock protein 27 is associated with decreased survival in node-negative breast cancer patients. Anticancer Res 25: 1649-1653, 2005.

40. Geisler JP, Geisler HE, Tammela J, Miller GA, Wiemann MC and Zhou Z: A study of heat shock protein 27 in endometrial carcinoma. Gynecol Oncol 72: 347-350, 1999.

41. Lo Muzio L, Campisi G, Farina A, et al: Prognostic value of HSP27 in head and neck squamous cell carcinoma: a retrospective analysis of 57 tumours. Anticancer Res 26: 1343-1349, 2006.

42. Malusecka E, Krzyzowska-Gruca S, Gawrychowski J, FiszerKierzkowska A, Kolosza Z and Krawczyk Z: Stress proteins HSP27 and HSP70i predict survival in non-small cell lung carcinoma. Anticancer Res 28: 501-506, 2008

43. Piura B, Rabinovich A, Yavelsky V and Wolfson M: [Heat shock proteins and malignancies of the female genital tract]. Harefuah 141: 969-1010, 2002.

44. Wang A, Liu X, Sheng S, et al: Dysregulation of heat shock protein 27 expression in oral tongue squamous cell carcinoma. BMC Cancer 9: 167, 2009.

45. Zanini C, Pulera F, Carta F, et al: Proteomic identification of heat shock protein 27 as a differentiation and prognostic marker in neuroblastoma but not in Ewing's sarcoma. Virchows Arch 452: 157-167, 2008.

46. Mineva I, Gartner W, Hauser P, et al: Differential expression of alphaB-crystallin and Hsp27-1 in anaplastic thyroid carcinomas because of tumor-specific alphaB-crystallin gene (CRYAB) silencing. Cell Stress Chaperones 10: 171-184, 2005.

47. Neininger A and Gaestel M: Evidence for a hsp25-specific mechanism involved in transcriptional activation by heat shock. Exp Cell Res 242: 285-293, 1998.

48. Yamashita K, Kim MS, Park HL, et al: HOP/OB1/NECC1 promoter DNA is frequently hypermethylated and involved in tumorigenic ability in esophageal squamous cell carcinoma. Mol Cancer Res 6: 31-41, 2008. 